This document is the accepted manuscript version of the following article: oliva, M. A., Prota, A. E., Rodríguez-Salarichs, J., Bennani, Y. L., Jiménez-Barbero, J., Bargsten, K., ... Díaz, J. F. (2020). Structural basis of noscapine activation for tubulin binding. Journal of Medicinal Chemistry, 63(15), 8495-8501. https://doi.org/10.1021/acs.jmedchem.0c00855

\title{
Structural basis of noscapine activation for tubulin binding
}

\author{
María A. Oliva ${ }^{1 \ddagger}$, Andrea E. Prota ${ }^{2 \ddagger}$, Javier Rodríguez-Salarichs ${ }^{1 \ddagger}$, Wenxin $\mathrm{Gu}^{3}$, Youssef L. Bennani ${ }^{4}$, \\ Jesús Jiménez-Barbero ${ }^{5,6,7}$, Katja Bargsten ${ }^{2 \dagger}$, Ángeles Canales ${ }^{8}$, Michel O. Steinmetz ${ }^{2,9}$, J. Fernando \\ Díaz $^{1 *}$
}

${ }^{1}$ Centro de Investigaciones Biológicas Margarita Salas-CSIC, Ramiro de Maeztu, 9, C.P. 28040, Madrid, Spain. ${ }^{2}$ Laboratory of Biomolecular Research, Division of Biology and Chemistry, Paul Scherrer Institut, CH-5232 Villigen PSI, Switzerland. ${ }^{3}$ Agios Pharmaceuticals, Inc. 88 Sidney Street Cambridge, MA 02139 United States. ${ }^{4}$ adMare Bio Innovations, Centre d'innovation NÉOMED, 7171 Frederick-Banting, Montréal, QCH4S 1z9, Canada. ${ }^{5}$ CIC bioGUNE, Basque Research Technology Alliance (BRTA), Bizkaia Technology Park, Building 801A, 48170 Derio, Spain. ${ }^{6}$ Ikerbasque, Basque Foundation for Science, María Díaz de Haro 13, 48009 Bilbao, Spain. ${ }^{7}$ Department of Organic Chemistry II, Faculty of Science \& Technology, University of the Basque Country, 48940 Leioa, Bizkaia, Spain. ${ }^{8}$ Dep. Química Orgánica I, Fac. Ciencias Químicas, Univ. Complutense de Madrid, Avd. Complutense s/n, C.P. 28040 Madrid, Spain. ${ }^{9}$ University of Basel, Biozentrum, CH-4056 Basel, Switzerland.

KEYWORDS: Noscapine, tubulin, microtubules, colchicine, anticancer drug.

ABSTRACT: Noscapine is a natural alkaloid that is used as an antitussive medicine. However, it also acts as a weak anticancer agent in certain in vivo models through a mechanism that is largely unknown. Here, we performed structural studies and show that the cytotoxic agent 7A-O-demethoxy-amino-noscapine (7A-aminonoscapine) binds to the colchicine site of tubulin. We suggest that the 7A-methoxy group of noscapine prevents binding to tubulin due to a steric clash of the compound with the T5-loop of $\alpha$-tubulin. We further propose that the anticancer activity of noscapine arises from a bioactive metabolite that binds to the colchicine site of tubulin to induce mitotic arrest through a microtubule cytoskeleton-based mechanism.

\section{Introduction}

Among systemic and wide spectrum anticancer chemotherapies, those directed against tubulin have shown to be extremely successful. Vinca drugs discovered in the 60 's were the first magic bullet against leukemias ${ }^{1}$, while taxanes became in the $90^{\prime} \mathrm{s}$ the best choice for the treatment of solid tumors (breast, ovarian, prostatic, lung $)^{2}$. More recently, high affinity drugs targeting the vinblastine site on $\beta$-tubulin (e.g., auristatin) or the newly discovered maytansine site ${ }^{3}$ had shown excellent activity as antibody drug conjugates $^{4}$. Finally, newly developed chemotypes with improved properties targeting the vinca (eribulin) ${ }^{5}$ or the taxane (ixabepilone) ${ }^{6}$ sites have been approved as second line treatments of metastatic cancers. However, no drug binding to the colchicine site has passed yet the trials required to become an approved anticancer drug, though this was the first druggable site described in tubulin ${ }^{7}$. Colchicine itself and the colchicine-site ligand mebendazole are currently the only drugs used for the treatment of gout ${ }^{8}$ and nematode infestation ${ }^{9}$, respectively.

Noscapine is a naturally occurring phthalideisoquinoline alkaloid obtained during opium harvesting ${ }^{10}$. It is used in humans as an oral antitussive agent ${ }^{11}$. The drug also displays a favorable toxicity profile and has been known for some time to act as a weak anticancer agent in certain in vivo models ${ }^{12}$. However, its molecular mechanism of action in this context is unknown. Like other microtubule targeting agents, noscapine arrests cells in the G2/M phase of the cycle at elevated concentrations $(2 \mu \mathrm{M})$, but it does not significantly alter microtubule formation in vitro ${ }^{12,13}$. Accordingly, the cellular effect could be caused by small amounts of an active contaminant or by metabolic activation of a prodrug. Strikingly, 7A O- demethylated noscapine-derivatives (7A-hydroxy and -amino derivatives) mirror the cellular phenotype of the parent molecule, but induce a G2/M arrest at about 500 times increased potency ${ }^{14}$. In a previous study, we suspected that the $7 \mathrm{~A}$-amino derivative of noscapine (7A-aminonoscapine) binds to the colchicine site of tubulin $^{13}$. Tubulin inhibition through the colchicine site is a substoichiometric process ${ }^{15}$ and involves blocking of the "curved to straight" conformational transition required for tubulin dimers to incorporate into the microtubule lattice ${ }^{16}$.

In this work, we performed an NMR-assisted docking process combining STD data and CORCEMA-ST calculations for the building of a 3D model that we further validated through the determination of the structure of the tubulin-7A-aminonoscapine complex bymacromolecular crystallography. Our results enlighten the molecular mechanism of action of noscapine by suggesting that noscapine is a prodrug that requires an activation step via O-demethylation at its position $7 \mathrm{~A}$

\section{Results}

NMR-directed modeling of the tubulin-7A-aminonoscapine complex in solution indicates binding to the colchicine site.

STD-NMR is a useful tool to investigate the interaction of small ligands with macromolecules. Aiming at understanding the lack of activity of noscapine on microtubule assembly and at unveiling the binding mode of 7A-aminonoscapine (Figure 1), we employed STD-NMR in combination with computational modelling. We further used the NMR STD and TR-NOESY data obtained previously ${ }^{13}$ to model binding poses of $7 \mathrm{~A}$-aminonoscapine in the two 

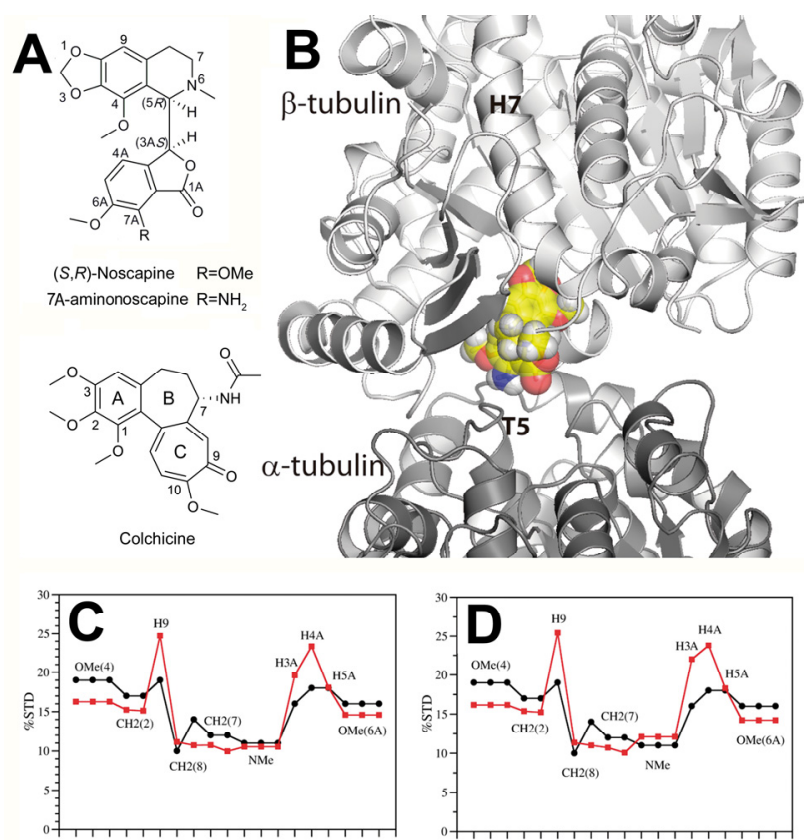

Figure 1.- A. Chemical structures of noscapine, 7A-aminonoscapine and colchicine. B. NMR calculated binding pose of 7A-aminonoscapine (yellow) at the colchicine site of $\beta 2$-tubulin of the $T_{2} R$ complex. C, D. Experimental STD profiles (black line) of the tubulin-7A-aminonoscapine complex as compared with STD profiles calculated (red line) from the generated binding poses in the $\beta 1$ (C) and $\beta 2$-tubulin (D) subunits in the $\mathrm{T}_{2} \mathrm{R}$-TTL complex.

tubulin dimers present in the $\mathrm{T}_{2} \mathrm{R}$-podophyllotoxin complex (PDB $1 \mathrm{SA} 1)^{16}$.

In agreement with the observed drug competition with the colchicine-site ligand nocodazole ${ }^{13}$, the best binding poses with similar scores located to the colchicine sites of both tubulin dimers in the $\mathrm{T}_{2} \mathrm{R}$ complex $(\beta 1$-tubulin $=0.16, \beta 2$-tubulin $=0.18$; Figure $1 \mathrm{~B})$. In both cases, nearly identical calculated STD profiles with r.m.s.d. values below $2 \AA$ between them were revealed (Figure $1 \mathrm{C}$-D). The calculated profiles were fully compatible with those experimentally determined, indicating that protons $\mathrm{H} 9, \mathrm{H} 3 \mathrm{~A}$ and $\mathrm{H} 4 \mathrm{~A}$ of the ligand are closer to the protein surface and thus receive a larger saturation.

High-resolution crystal structure of the $T_{2} R-T T L-7 A-$ aminonoscapine complex.

Despite noscapine had shown a weak colchicine-like activity, we were previously unable to detect any direct binding of the compound to the colchicine site using centrifugation- or spectroscopicbased assays ${ }^{13}$. Thus, the reason for the observed antitubulin activity of noscapine remains unknown.

Both NMR determined poses (i.e., in $\beta 1$ - and $\beta 2$-tubulin of the $T_{2} R$ complex) revealed 7A-aminoscapine in the same orientation, but its placement in the two binding sites is shifted with respect to each other (Figure S1), suggesting that the method does not have enough accuracy to understand the reason for noscapine's inability to bind the colchicine site. In order to investigate the structural reasons for the lack of activity of noscapine, we performed soaking experiments with noscapine and 7A-aminonoscapine using $\mathrm{T}_{2} \mathrm{R}$-TTL crystals obtained from a protein complex composed of two $\alpha \beta$-tubulin heterodimers, the stathmin-like protein RB3 and tubulin tyrosine ligase ${ }^{17,18}$. The $\mathrm{T}_{2} \mathrm{R}$-TTL system has successfully produced
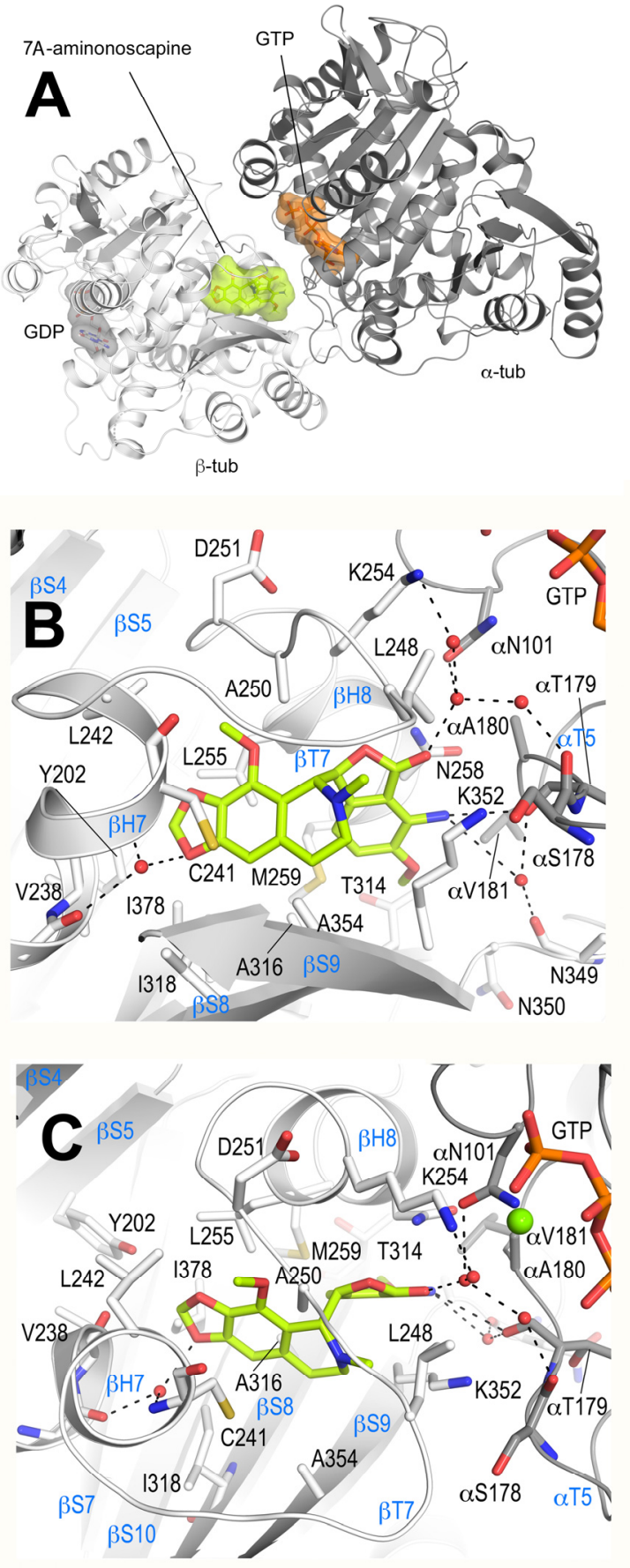

Figure 2.- Overall tubulin-7A-aminonoscapine complex structure. A. Ribbon representation of the tubulin-bound 7A-aminonoscapine structure (PDB ID 6Y6D). The $\alpha$ - and $\beta$-tubulin chains are in dark and light grey, respectively. The ligand 7A-aminonoscapine (light green) and the nucleotides (orange) are in sphere and stick representation, respectively. The carbon atoms of the individual nucleotides are colored according to their chain assignments. Oxygen and nitrogen atoms are colored in red and blue, respectively. B, C. Close-up view of the atomic interaction network observed between 7A-aminonoscapine (light green) and tubulin (gray) in two different orientations. Interacting residues of tubulin are shown in stick representation and are labeled. Oxygen and nitrogen atoms are colored red and blue, respectively. Hydrogen bonds are depicted as black dashed lines. Secondary structural elements of tubulin are labeled in blue. For simplicity, only $\alpha$-tubulin residues are indicated with a " $\alpha$ ". 


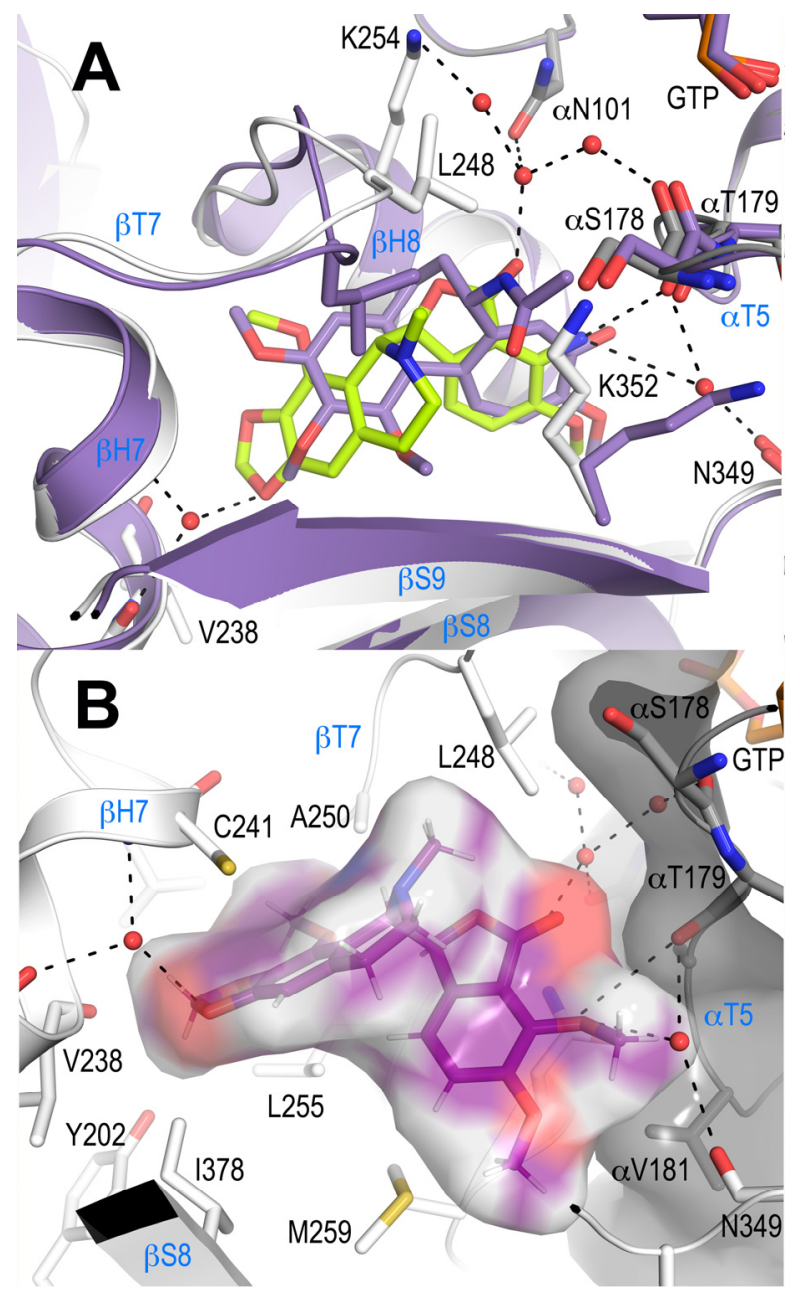

Figure 3.- A. Superposition of the tubulin-7A-aminonoscapine (light green, PDB ID 6Y6D) and tubulin-colchicine (slate, PDB ID 4O2B) complex structures. The structures were superimposed onto the $\beta 1$-tubulin of their respective T2R-TTL complexes. Interacting residues of tubulin discussed in the text are shown in stick representation and are labeled. Oxygen and nitrogen atoms are colored red and blue, respectively. Hydrogen bonds are depicted as black dashed lines. Secondary structural elements of tubulin are labeled in blue. B. Modeled noscapine molecule into the crystallographic determined 7A-aminonoscapine binding site, highlighting how the 7A-methoxy group would clash into the $\alpha$ T5-loop. The clash is in agreement with the calculated NMR-model and suggests why noscapine does not bind to tubulin.

several structures of ligands bound to tubulin using X-ray crystallography (reviewed $\mathrm{in}^{19}$ ). The structure of $\mathrm{T}_{2} \mathrm{R}-\mathrm{TTL}-7 \mathrm{~A}-$ aminonoscapine was solved at $2.2 \AA$ resolution and we unequivocally found ligand density at the colchicine site (Figures 2A, S2). Concomitantly with the biochemical experiments ${ }^{13}$, we could not identify any extra density from crystals soaked with the parental noscapine (not shown).

The ligand in the colchicine site occupies a similar space as colchicine (Figure 3A). Noticeably, the conformation of 7A-aminonoscapine using either NMR or crystallography is similar with an r.m.s.d. of $0.18 \AA$ in $\beta 2$-tubulin (Figure S1A) while the NMR calculated pose is displaced from the actual crystallographic one in $\beta 1$-tubulin (Figure S1B). The overall structure of tubulin in the $\mathrm{T}_{2} \mathrm{R}$-TTL-7A-aminonoscapine complex superimposed well with that of the ligand free complex structure ${ }^{18}$ with an overall r.m.s.d. of $0.29 \AA$ over $1996 \mathrm{C}_{\alpha}$-atoms and a $\beta$-tubulin r.m.s.d. of $0.14 \AA$ over $365 \mathrm{C}_{\alpha}$-atoms, suggesting that the binding of $7 \mathrm{~A}$-aminonoscapine does not affect the conformation of tubulin.

The 3-membered $[1,3]$ dioxolo-isoquinoline ring of $7 \mathrm{~A}$-aminonoscapine is stacked between the side chains of $\beta$ Cys 241 of helix $\beta \mathrm{H} 7$ and $\beta$ Leu 255 of helix $\beta \mathrm{H} 8$ (Figures 3B-C). The dioxolo moiety points into the pocket shaped by the side chains of $\beta$ Tyr202, $\beta$ Val238, $\beta$ Leu242, $\beta$ Leu255, $\beta$ Ile318 and $\beta$ Ile 378, and the oxygen $\mathrm{O} 1$ forms two water-mediated $\mathrm{H}$-bonds to both the main chain carbonyl of $\beta$ Gly 237 and the main chain amide of $\beta$ Cys 241 of helix $\beta$ H7. The N6-methyl group is equatorially oriented towards the nucleotide $\mathrm{N}$-site of $\alpha$-tubulin, however, it does not engage in electrostatic interactions with the nucleotide. The protonated N6 points towards the sidechain of $\beta C y s 241$ and may engage in a weak interaction with the lone pairs of the sulfhydryl group (4.9 $\AA$ distance). The 4-methoxy moiety forms a hydrophobic contact with the side chains of $\beta$ Leu 242 and $\beta A$ la250. The benzofuran-1-one moiety is stacked between the side chains of $\beta$ Asn 258 and $\beta$ Lys 352 , and its 1A-carbonyl forms water-mediated $\mathrm{H}$-bonds to the side chains of $\alpha$ Asn 101 and $\beta$ Lys 254 and to the main chain carbonyl of $\alpha \operatorname{Ser} 178$. The 7A-amino group points towards the tubulin intradimer interface and is in direct and water mediated H-bond contact to both the main chain carbonyls of $\alpha$ Thr 179 and $\beta$ Asn 349 . The 6 A-methoxymoiety occupies the cavity shaped by the carboxy-terminal end of helix $\beta \mathrm{H} 8$, the amino-terminal end of strand $\beta \mathrm{S} 9$ and the $\alpha$ Val181 side chain on the $\alpha \mathrm{T} 5$-loop.

The tubulin-colchicine and tubulin-7A-aminonoscapine structures superimpose very well (Figure 4; overall r.m.s.d. of $0.16 \AA$ over $1882 \mathrm{C}_{\alpha}$-atoms and a $\beta$-tubulin r.m.s.d. of $0.14 \AA$ over $328 \mathrm{C}_{\alpha}$-atoms). The 7A-amino and the 6A-methoxy moieties of the benzofuran-1-one ring occupy almost the same space as the corresponding carbonyl and methoxy groups of the C-ring of colchicine, thereby establishing similar polar and hydrophobic contacts. Compared to both the colchicine-bound and the apo tubulin state, the equatorial N6-methyl group of 7A-aminonoscapine displaces the side chain of $\beta$ Leu247 thereby causing a flip of the $\beta$ T7-loop. The $\beta$ Lys 352 side chain is flipped towards the ligand and occupies the space that is otherwise filled by the 7-acetamide moiety of colchicine. Overall, these structural similarities suggest that both ligands most likely perturb tubulin by a similar mechanism, i.e., by preventing the curved to straight conformational transition required for tubulin assembly into microtubules ${ }^{20}$.

\section{Discussion and Conclusions}

Our high-resolution crystal structure confirms that 7A-aminonoscapine targets the colchicine site of tubulin (Figure 2). This result corroborates that the inhibitory effects of noscapine derivatives on tubulin assembly into microtubules and the subsequent cellular effects $^{13,14}$ are exerted using the same molecular mechanism as colchicine. Based on our structural information, we can now explain the lack of noscapine's activity in vitro and the activity improvement of some developed derivatives. The 7A-methoxy group in noscapine clashes with the $\alpha$ T5 loop of tubulin, precluding noscapine binding to the colchicine site (Figure $3 \mathrm{~B}$ ). The reduction of the noscapine lactone produced cyclic ether noscapinoids with increased activity ${ }^{21}$, which could be explained by the loss of the Hbond network established by the 1A-carbonyl group with $\alpha$-tubulin; it would also allow for a better accommodation of the 7A-methoxy group within the pocket. The best cellular toxicity activities were obtained with 9-halogenated derivatives ${ }^{22}$, which likely display increased contacts with strands S8-S9 in $\beta$-tubulin. Otherwise, $N$-substituted 6 -derivatives showed different effects on the inhibi- 
tion of cell proliferation ${ }^{21}$, which could be explained by the interaction of the different moieties with the $\beta$ T7-loop. Finally, bulkier substitutes at the 7A-position showed better activities than noscapine $^{23}$. The most reasonable explanation for those results is that derivatives containing such substitutes bind tubulin in a different way. An immediate question emerging from these observations is the following: from where does the colchicine-like activity observed for the parental noscapine come from? We hypothesize that the observed in vivo activity of noscapine is the result of its metabolic bioactivation. A prodrug is a molecule with a low or neglectable activity against a pharmacological target as compared with one of its metabolites ${ }^{24}$. In humans, the metabolism of xenobiotics is mostly related with cytochrome P450 enzymes that among other activities perform the oxidation of a carbon group bound to oxygen or other heteroatoms ${ }^{25}$. This reaction leads to the release of the carbon and leaves an alcohol, amine or thiol in the molecule ${ }^{25}$, which is a common mechanism for the release of a drug from its prodrug $^{24}$. For instance, morphine or norcodeine are the consequence of O-demethylation and N-demethylation of codein, respectively ${ }^{26}$. In fact, the oxidation mechanism is a common way of antitumoral prodrug activation (e.g., cyclophosphamide ${ }^{27}$, dacarbazine ${ }^{28}$, tamoxifen ${ }^{29}$ ), where the hepatic CYP2D6 enzyme is mostly involved in the activation process, as exemplified by codeine and tamoxi$\mathrm{fen}^{24}$.

The noscapine metabolism is well understood and includes extensive 'first pass' metabolism in rats, rabbits and humans mainly by $\mathrm{C}-\mathrm{C}$ cleavage, $\mathrm{O}$-demethylation and cleavage of the methylenedioxyl group ${ }^{30-32}$. The metabolic map of noscapine has been extensively characterized by Fang et $\mathrm{al}^{32}$. This study shows that the 7Ademethylated product is one of the major metabolites of noscapine in cells and in vivo after being processed by CYP3A5. The same metabolite was also identified in mice both unmodified and as its glucuronide after being processed by UGT1A1/3/9. The 7A-demethylated product of noscapine is a tubulin assembly inhibitor that is as potent as vinblastine, showing an IC50 of $0.6 \mu \mathrm{M}$ in A549 cells ${ }^{14}$. Considering that the noscapine doses effective for tumour inhibition in mice are $120 \mathrm{mg} / \mathrm{kg}^{12}$, while the dose of colchicine (when used as antigout drug) is about 4000 times lower (i.e 30 $\mu \mathrm{g} / \mathrm{kg}^{33}$ ), it is reasonable to assume that only a small fraction of the metabolically processed 7A-desmethylated noscapine derivative is required to justify the observed activity.

In conclusion, we propose that noscapine's cytotoxicity is a consequence of the oxidation of its methoxy carbon at position 7A, which leads to the formation of the active phenol. The resulting drug is then able to bind tubulin and block microtubule assembly in a colchicine-like manner ${ }^{13}$.

\section{Experimental Section.}

Proteins and ligands.

Purified calf brain tubulin and chemicals were obtained as previously described $^{34,35}$. Noscapine was from Merck. 7A-aminonoscapine (Figure 1) was synthesized as described ${ }^{14}$. The compounds were diluted in $99.8 \%$ D6-DMSO (Merck) to a final concentration of $20 \mathrm{mM}$ and stored at $-20^{\circ} \mathrm{C}$.

\section{NMR directed structure modeling.}

The structure of the 7A-aminonoscapine-tubulin complex was modeled using molecular docking based on NMR-STD measurements ${ }^{36}$. We employed the NMR-STD spectra of 7A-aminonoscapine bound to tubulin previously obtained, the coordinates of the 7A-aminonoscapine bound to the protein (obtained from TR-NOESY measurements) ${ }^{13}$ and the structure of tubulin in the $\mathrm{T}_{2} \mathrm{R}$-podophyllotoxin-tubulin complex (pdb:1SA1) ${ }^{16}$ after removing podophyllotoxin from the binding site. Prior to use, the structures were optimized using Polak-Ribiere conjugated gradient (PRCG) and implemented in Macromodel ${ }^{37}$ employing the OPLS2005 force field ${ }^{38-40}$. Water solvation was implicitly considered following generalized Born model $\mathrm{GB} / \mathrm{SA}^{41}$. Cutoff distances for the van der Waals potential were $8.0 \AA$, while these for the electrostatic potential was $20.0 \AA$. The maximum number of iterations was set to 9000 while the minimization was considered finished when the energy gradient was smaller than $0.05 \mathrm{~kJ} / \mathrm{mol}^{-1}$.

We generated the complex structure by iterative fitting of the STD spectrum calculated from generated binding poses to the experimentally determined STD spectrum. To do so, we calculated tens of binding poses employing Autodock-Vina ${ }^{42}$ within a defined area of the protein surface, which was considered rigid. In this work, a $25 \AA$ parallelepiped build around $\beta 1$ - and $\beta 2$-tubulin-cavities was employed, and 20 poses were generated per cavity. The theoretical STD spectrum of 7A-aminoscapine bound to tubulin was calculated for each binding pose using the CORCEMA-ST matlab rutine ${ }^{43,44}$. For these full relaxation matrix calculations, an order parameter $\mathrm{S}^{2}=0.85$ was employed to account for the fast rotation of the methyl groups, as implemented in CORCEMAST. For each generated pose the apparent binding and association constants $\left(\mathrm{K}_{\mathrm{b}}^{\mathrm{app}}, \mathrm{k}_{\mathrm{on}}{ }^{\mathrm{app}}\right)$ and the rotational correlation time of the free $\tau_{\mathrm{c}}{ }_{\mathrm{c}}^{\text {fapp }}$ and bound $\tau_{\mathrm{c}}$ bapp compound were optimized to minimize the difference between the calculated and the measured STD spectrum. The best binding poses were selected and sorted according to their difference with the experimental STD spectrum. The structure with the best score was found in the colchicine site employing the following parameters $\mathrm{Kb}_{\mathrm{b}}^{\text {app }}$ $1 \pm 0.5 \times 10^{5} \mathrm{M}^{-1}, \mathrm{k}_{\mathrm{on}}{ }^{\text {app }} 1 \pm 0.5 \times 10^{6} \mathrm{M}^{-1} \mathrm{~s}^{-1} \tau_{\mathrm{c}}^{\mathrm{f}}{ }^{\mathrm{f}, \mathrm{ppp}} 0.8 \pm 0.3 \mathrm{~ns}, \tau_{\mathrm{c}}^{\text {b,app }} 100 \pm 50$ ns (for site $\beta 1$ ) or $80 \pm 5$ ns (for site $\beta 2$ ) ns.

Crystallization, Data Collection, and Structure Determination Crystals of $\mathrm{T}_{2} \mathrm{R}$-TTL were grown as described by Prota et al. ${ }^{17,18}$. Briefly, the $T_{2} R-T T L$ complex was crystallized by the vapor-diffusion method at $20^{\circ} \mathrm{C}$ using the micro-seeding technique. Crystals grew over two days in precipitant solution consisting of $5 \%$ PEG $4 \mathrm{~K}, 11 \%$ glycerol, $30 \mathrm{mM} \mathrm{MgCl}_{2}, 30 \mathrm{mM} \mathrm{CaCl}_{2}, 100 \mathrm{mM}$ MES/Imidazole, $\mathrm{pH} 6.5$. The crystals were soaked over-night at $20^{\circ} \mathrm{C}$ in reservoir solutions containing $2 \mathrm{mM}$ of 7A-aminonoscapine, and subsequently flash-cooled in liquid nitrogen upon two consecutive transfers to reservoir solutions containing i) $10 \%$ PEG $4 \mathrm{~K}$ and $16 \%$ glycerol, and ii) $10 \%$ PEG $4 \mathrm{~K}$ and $20 \%$ glycerol, respectively. Native data were collected at $100 \mathrm{~K}$ at beamline X06DA of the Swiss Light Source (Paul Scherrer Institut, Villigen PSI, Switzerland). Data were processed and merged with $\mathrm{XDS}^{45}$ to $2.2 \AA$ resolution.

The $T_{2} R$-TTL-7A-aminonoscapine structure was determined by the difference Fourier method using the phases of the $T_{2} R$-TTL complex (PDB ID 4I4T) in the absence of ligands and solvent molecules as a starting point for refinement. After three cycles of rigid body and subsequent three cycles of restrained refinement in Phenix ${ }^{46}$, clear difference density delineating the shape of a bound molecule to the colchicine site was visible.

After correction of geometry, Ramachandran and rotamer outliers, missing water molecules were added to the model, and additional cycles of restrained refinement in Phenix ${ }^{46}$ were performed. The 7A-aminonoscapine molecule was then placed into the difference electron density map at the colchicine site and the model was further improved by iterative rounds of model building and refinement. The final model was refined to $2.2 \AA$ resolution with crystallographic $\mathrm{R}$ values of $18.4 \%$ $\left(\mathrm{R}_{\text {work }}\right)$ and $22.4 \%\left(\mathrm{R}_{\text {free}}\right)$, respectively. The model has good geometry with small root mean square deviations (rmsd) from ideal values for bond lengths and bond angles. The quality of the structures was assessed with MolProbity ${ }^{47}$. Data collection and refinement statistics are given in Table S1. Molecular graphics and analyses were performed with PyMol (The PyMOL Molecular Graphics System, Version 1.8.6.2. Schrödinger, LLC).

\section{ASSOCIATED CONTENT}

\section{Supporting Information}

This material is available free of charge via the Internet at http://pubs.acs.org. 
Table S1. Data collection and refinement statistics of the crystallographic analysis Figure S1. Comparison between crystal and NMR structures. Figure S2. Omit map of tubulin-bound 7A-aminonoscapine (pdf file). NMR models of the T2R-TTL-7demethoxy-amino noscapine complexes (pdb files). Molecular Formula Strings.

\section{PDB codes}

PDB code for the crystallographic structure of the $\mathrm{T}_{2} \mathrm{R}-\mathrm{TTL}-7-$ demethoxy-amino noscapine complex is 6Y6D.

\section{AUTHOR INFORMATION}

\section{Corresponding Author}

*E-mail: fer@cib.csic.es

\section{Present Addresses}

$\uparrow$ Current address: Department of Biochemistry, University of Zürich Winterthurerstrasse 190, 8057 Zürich (Switzerland).

\section{Author Contributions}

$\$$ These authors contributed equally.

\section{ACKNOWLEDGMENT}

We thank Ganadería Fernando Díaz for calf brains supply and staff of beamlines X06DA of the Swiss Light Source (Paul Scherrer Institut, Villigen PSI, Switzerland) for their support. This work was supported by Ministerio de Ciencia e Innovación PID2019104545RB-I00 and Fondo de Investigaciones Sanitarias COV2001007 to JFD; CTQ2016-76263-P to AC; Swiss National Science Foundation (31003A_166608) to MOS; H2020-MSCA-ITN-2019 860070 TUBINTRAIN to AEP and JFD and a donation from Club deportivo Escuela Hungaresa de Pontevedra.

\section{ABBREVIATIONS}

CORCEMA-ST Complete relaxation and conformational exchange matrix analysis of saturation transfer; STD Saturation Transfer Difference, TTL- $\mathrm{T}_{2} \mathrm{R}$ bovine brain $\alpha \beta$-tubulin, rat stathmin-like protein RB3, and chicken tubulin-tyrosine ligase complex; TRNOESY Two-dimensional transferred nuclear Overhauser effect spectroscopy.

\section{REFERENCES}

1. Johnson, I. S.; Armstrong, J. G.; Gorman, M.; Burnett, J. P. The Vinca Alkaloids: A New Class of Oncolytic Agents. Cancer Res. 1963, 23, 1390-1427.

$2 . \quad$ Donehower, R. C. The Clinical Development of Paclitaxel: A Successful Collaboration of Academia, Industry and the National Cancer Institute. Oncologist 1996, 1, 240-243.

3. Prota, A. E.; Bargsten, K.; Diaz, J. F.; Marsh, M.; Cuevas, C.; Liniger, M.; Neuhaus, C.; Andreu, J. M.; Altmann, K. H.; Steinmetz, M. O. A new Tubulin-binding Site and Pharmacophore for Microtubule-destabilizing Anticancer Drugs. Proc Natl Acad Sci U S A 2014, 111, 13817-13821.

4. Lambert, J. M.; Chari, R. V. J. Ado-trastuzumab Emtansine (T-DM1): An Antibody-Drug Conjugate (ADC) for HER2-Positive Breast Cancer. J. Med. Chem. 2014, 57, 6949-6964.

5. Doodhi, H.; Prota, A. E.; Rodriguez-Garcia, R.; Xiao, H.; Custar, D. W.; Bargsten, K.; Katrukha, E. A.; Hilbert, M.; Hua, S.; Jiang, K.; Grigoriev, I.; Yang, C. H.; Cox, D.; Horwitz, S. B.; Kapitein, L. C.; Akhmanova, A.; Steinmetz, M. O. Termination of Protofilament Elongation by Eribulin Induces Lattice Defects that Promote Microtubule Catastrophes. Curr Biol 2016, 26, 1713-1721.

$6 . \quad$ Denduluri, N.; Swain, S. M. Ixabepilone for the Treatment of Solid Tumors: a Review of Clinical Data. Expert Opin Investig Drugs 2008, 17, 423-435.
7. Borisy, G. G.; Taylor, E. W. The Mechanism of Action of Colchicine. Binding of Colchincine-3H to Cellular Protein. J Cell Biol 1967, 34, 525-533.

8. Khanna, D.; Khanna, P. P.; Fitzgerald, J. D.; Singh, M. K.; Bae, S.; Neogi, T.; Pillinger, M. H.; Merill, J.; Lee, S.; Prakash, S.; Kaldas, M.; Gogia, M.; Perez-Ruiz, F.; Taylor, W.; Lioté, F.; Choi, H.; Singh, J. A.; Dalbeth, N.; Kaplan, S.; Niyyar, V.; Jones, D.; Yarows, S. A.; Roessler, B.; Kerr, G.; King, C.; Levy, G.; Furst, D. E.; Edwards, N. L.; Mandell, B.; Schumacher, H. R.; Robbins, M.; Wenger, N.; Terkeltaub, R.; American College of, R. 2012 American College of Rheumatology Guidelines for Management of Gout. Part 2: Therapy and Antiinflammatory Prophylaxis of Acute Gouty Arthritis. Arthritis Care Res (Hoboken) 2012, 64, 1447-1461.

9. Lacey, E. Mode of Action of Benzimidazoles. Parasitol. Today 1990, 6, 112-115.

10. Warolin, C. Pierre-Jean Robiquet (Rennes, 14 janvier 1780Paris, 29 avril 1840). Rev. Hist. Pharm. 1999, 97-110.

11. Harpal, S.; Prashant, S.; Kamlesh, K.; Ankush, C.; Sujata, K. D.; Ramesh, C. A Review on Noscapine, and its Impact on Heme Metabolism. Curr. Drug Metab. 2013, 14, 351-360.

12. Ye, K.; Ke, Y.; Keshava, N.; Shanks, J.; Kapp, J. A.; Tekmal, R. R.; Petros, J.; Joshi, H. C. Opium Alkaloid Noscapine is an Antitumor Agent that Arrests Metaphase and induces Apoptosis in Dividing Cells. Proc Natl Acad Sci U S A 1998, 95, 1601-1606.

13. Bennani, Y. L.; Gu, W.; Canales, A.; Díaz, J. F.; Eustace, B. K.; Hoover, R. R.; Jiménez-Barbero, J.; Nezami, A.; Wang, T. Tubulin Binding, Protein-Bound Conformation in Solution, and Antimitotic Cellular Profiling of Noscapine and Its Derivatives. J. Med. Chem. 2012, 55, 1920-1925.

14. Anderson, J. T.; Ting, A. E.; Boozer, S.; Brunden, K. R.; Crumrine, C.; Danzig, J.; Dent, T.; Faga, L.; Harrington, J. J.; Hodnick, W. F.; Murphy, S. M.; Pawlowski, G.; Perry, R.; Raber, A.; Rundlett, S. E.; Stricker-Krongrad, A.; Wang, J.; Bennani, Y. L. Identification of Novel and Improved Antimitotic Agents Derived from Noscapine. $J$. Med. Chem. 2005, 48, 7096-7098.

15. Perez-Ramirez, B.; Andreu, J. M.; Gorbunoff, M. J.; Timasheff, S. N. Stoichiometric and Substoichiometric Inhibition of Tubulin Self-Assembly by Colchicine Analogues. Biochemistry 1996, 35, 3277-85.

16. Ravelli, R. B.; Gigant, B.; Curmi, P. A.; Jourdain, I.; Lachkar, S.; Sobel, A.; Knossow, M. Insight into Tubulin Regulation from a Complex with Colchicine and a Stathmin-like Domain. Nature 2004, 428, 198-202.

17. Prota, A. E.; Bargsten, K.; Zurwerra, D.; Field, J. J.; Diaz, J. F.; Altmann, K. H.; Steinmetz, M. O. Molecular Mechanism of Action of Microtubule-Stabilizing Anticancer Agents. Science 2013, 339, 587590 .

18. Prota, A. E.; Magiera, M. M.; Kuijpers, M.; Bargsten, K.; Frey, D.; Wieser, M.; Jaussi, R.; Hoogenraad, C. C.; Kammerer, R. A.; Janke, C.; Steinmetz, M. O. Structural Basis of Tubulin Tyrosination by Tubulin Tyrosine Ligase. J Cell Biol 2013, 200, 259-270.

19. Steinmetz, M. O.; Prota, A. E. Microtubule-Targeting Agents: Strategies To Hijack the Cytoskeleton. Trends Cell Biol 2018 , 776-792.

20. Buey, R. M.; Díaz, J. F.; Andreu, J. M. The Nucleotide Switch of Tubulin and Microtubule Assembly: a Polymerization-driven Structural Change. Biochemistry 2006, 45, 5933-5938.

21. DeBono, A. J.; Xie, J. H.; Ventura, S.; Pouton, C. W.; Capuano, B.; Scammells, P. J. Synthesis and Biological Evaluation of N-Substituted Noscapine Analogues. ChemMedChem 2012, 7, 21222133 .

22. Aneja, R.; Vangapandu, S. N.; Lopus, M.; Viswesarappa, V. G.; Dhiman, N.; Verma, A.; Chandra, R.; Panda, D.; Joshi, H. C. Synthesis of Microtubule-Interfering Halogenated Noscapine Analogs that perturb Mitosis in Cancer Cells followed by Cell Death. Biochem. Pharmacol. 2006, 72, 415-426.

23. Mishra, R. C.; Karna, P.; Gundala, S. R.; Pannu, V.; Stanton, R. A.; Gupta, K. K.; Robinson, M. H.; Lopus, M.; Wilson, L.; Henary, M.; Aneja, R. Second Generation Benzofuranone Ring substituted Noscapine Analogs: Synthesis and Biological Evaluation. Biochem. Pharmacol. 2011, 82, 110-121. 
24. Ortiz de Montellano, P. R. Cytochrome P450-activated Prodrugs. Future Med. Chem. 2013, 5, 213-228.

25. Anzenbacher, P.; Anzenbacherová, E. Cytochromes P450 and Metabolism of Xenobiotics. Cell. Mol. Life Sci. 2001, 58, 737-747. 26. Sindrup, S. H.; Brøsen, K. The Pharmacogenetics of Codeine Hypoalgesia. Pharmacogenet. Genom. 1995, 5, 335-346. 27. Friedman, O. M.; I., W.; Myles, A. Cyclophosphamide (NSC-26271)-related Phosphoramide Mustards- Recent Advances and Historical Perspective. Cancer Treat Rep 1976 60, 337-346.

28. Marchesi, F.; Turriziani, M.; Tortorelli, G.; Avvisati, G.; Torino, F.; De Vecchis, L. Triazene Compounds: Mechanism of Action and Related DNA Repair Systems. Pharmacol. Res. 2007, 56, 275-287. 29. Desta, Z.; Ward, B. A.; Soukhova, N. V.; Flockhart, D. A. Comprehensive Evaluation of Tamoxifen Sequential Biotransformation by the Human Cytochrome P450 System in Vitro: Prominent Roles for CYP3A and CYP2D6. J. Pharmacol. Exp. Ther 2004, 310, 1062-1075.

30. Tsunoda, N.; Yoshimura, H. Metabolic Fate of Noscapine.; III. Further Studies on Identification and Determination of the Metabolites. Xenobiotica 1981, 11, 23-32.

31. Tsunoda, N.; Yoshimura, H. Metabolic Fate of Noscapine. II. Isolation and identification of Novel Metabolites produced by $\mathrm{C}-$ C bond Cleavage. Xenobiotica 1979, 9, 181-187.

32. $\quad$ Fang, Z.-Z.; Krausz, K. W.; Li, F.; Cheng, J.; Tanaka, N.; Gonzalez, F. J. Metabolic Map and Bioactivation of the anti-Tumour Drug Noscapine. Br. J. Pharmacol 2012, 167, 1271-1286.

33. Slobodnick, A.; Shah, B.; Krasnokutsky, S.; Pillinger, M. H. Update on Colchicine, 2017. Rheumatology Oxford 2018, 57, i4-i11. 34. Díaz, J. F.; Andreu, J. M. Assembly of Purified GDPTubulin into Microtubules induced by Taxol and Taxotere: Reversibility, Ligand Stoichiometry, and Competition. Biochemistry 1993, 32, 2747-2755.

35. Andreu, J. M. Large Scale Purification of Brain Tubulin. In Methods Mol. Biol., Zhou, J., Ed. Humana Press Inc.: Totowa, NJ, 2007; Vol. 137, pp 17-28.

36. Rodríguez-Salarichs, J.; Canales, A.; Diaz, J. F. PhD Thesis: Desarrollo y Validación Experimental de un Método para la Obtención de Estructuras de Complejos Ligando/Receptor basado en Resonancia Magnética Nuclear. 2017.

37. Mohamadi, F.; Richards, N. G. J.; Guida, W. C.; Liskamp, R.; Lipton, M.; Caufield, C.; Chang, G.; Hendrickson, T.; Still, W. C. MacroModel an Integrated Software System for Modeling Organic and Bioorganic Molecules using Molecular Mechanics. J. Comput. Chem. 1990, 11, 440-467.

38. Jorgensen, W. L.; Tiradorives, J. The OPLS Potential Functions for Proteins - Energy Minimizations for Crystals of Cyclicpeptides and Crambin. . JACS 1988, 110, 1657-1666.
39. Kaminski, G. A.; Friesner, R. A.; Tirado-Rives, J.; Jorgensen, W. L. Evaluation and Reparametrization of the OPLS-AA Force Field for Proteins via Comparison with Accurate Quantum Chemical Calculations on Peptides. J. Phys. Chem. B 2001, 105, 64746487.

40. Bertelsen, L. B.; Shen, Y. Y.; Nielsen, T.; StodkildeJorgensen, H.; Lloyd, G. K.; Siemann, D. W.; Horsman, M. R. Vascular Effects of Plinabulin (NPI-2358) and the Influence on Tumour Response when given alone or combined with Radiation. Int $J$ Radiat Biol 2011, 87, 1126-1134.

41. Still, W. C.; Tempczyk, A.; Hawley, R. C.; Hendrickson, T. Semianalytical Treatment of Solvation for Molecular Mechanics and Dynamics. J Am Chem Soc 1990, 112, 6127-6129.

42. Trott, O.; Olson, A. J. AutoDock Vina: improving the Speed and Accuracy of Docking with a new Scoring Function, Efficient Optimization, and Multithreading. J. Comput. Chem. 2010, 31, 455461.

43. Moseley, H. N. B.; Curto, E. V.; Krishna, N. R. Complete Relaxation and Conformational Exchange Matrix (CORCEMA) Analysis of NOESY Spectra of Interacting Systems - 2-Dimensional Transferred NOESY. J Mag Reson Ser B 1995, 108, 243-261.

44. Jayalakshmi, V.; Krishna, N. R. Complete Relaxation and Conformational Exchange Matrix (CORCEMA) Analysis of Intermolecular Saturation Transfer Effects in Reversibly forming Ligand-Receptor Complexes. J Magn Reson 2002, 155, 106-118.

45. Kabsch, W. XDS. Acta Crystallogr. Sect. D 2010, 66, 125132.

46. Adams, P. D.; Afonine, P. V.; Bunkoczi, G.; Chen, V. B.; Davis, I. W.; Echols, N.; Headd, J. J.; Hung, L.-W.; Kapral, G. J.; Grosse-Kunstleve, R. W.; McCoy, A. J.; Moriarty, N. W.; Oeffner, R.; Read, R. J.; Richardson, D. C.; Richardson, J. S.; Terwilliger, T. C.; Zwart, P. H. PHENIX: a Comprehensive Python-based System for Macromolecular Structure Solution. Acta Crystallogr. Sect. D 2010, $66,213-221$.

47. Chen, V. B.; Arendall, W. B., III; Headd, J. J.; Keedy, D. A.; Immormino, R. M.; Kapral, G. J.; Murray, L. W.; Richardson, J. S.; Richardson, D. C. MolProbity: all-Atom Structure Validation for Macromolecular Crystallography. Acta Crystallogr. Sect. D 2010, 66, $12-21$ 


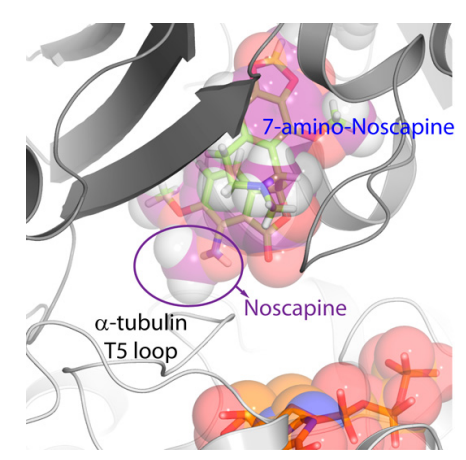




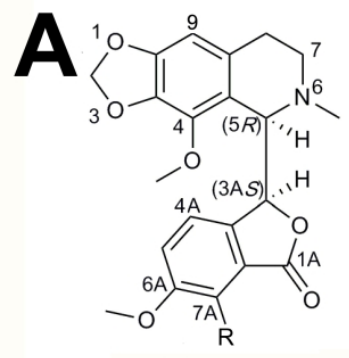

$(S, R)$-Noscapine $\quad \mathrm{R}=\mathrm{OMe}$ 7A-aminonoscapine $\mathrm{R}=\mathrm{NH}_{2}$

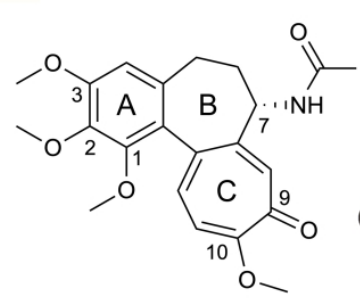

Colchicine
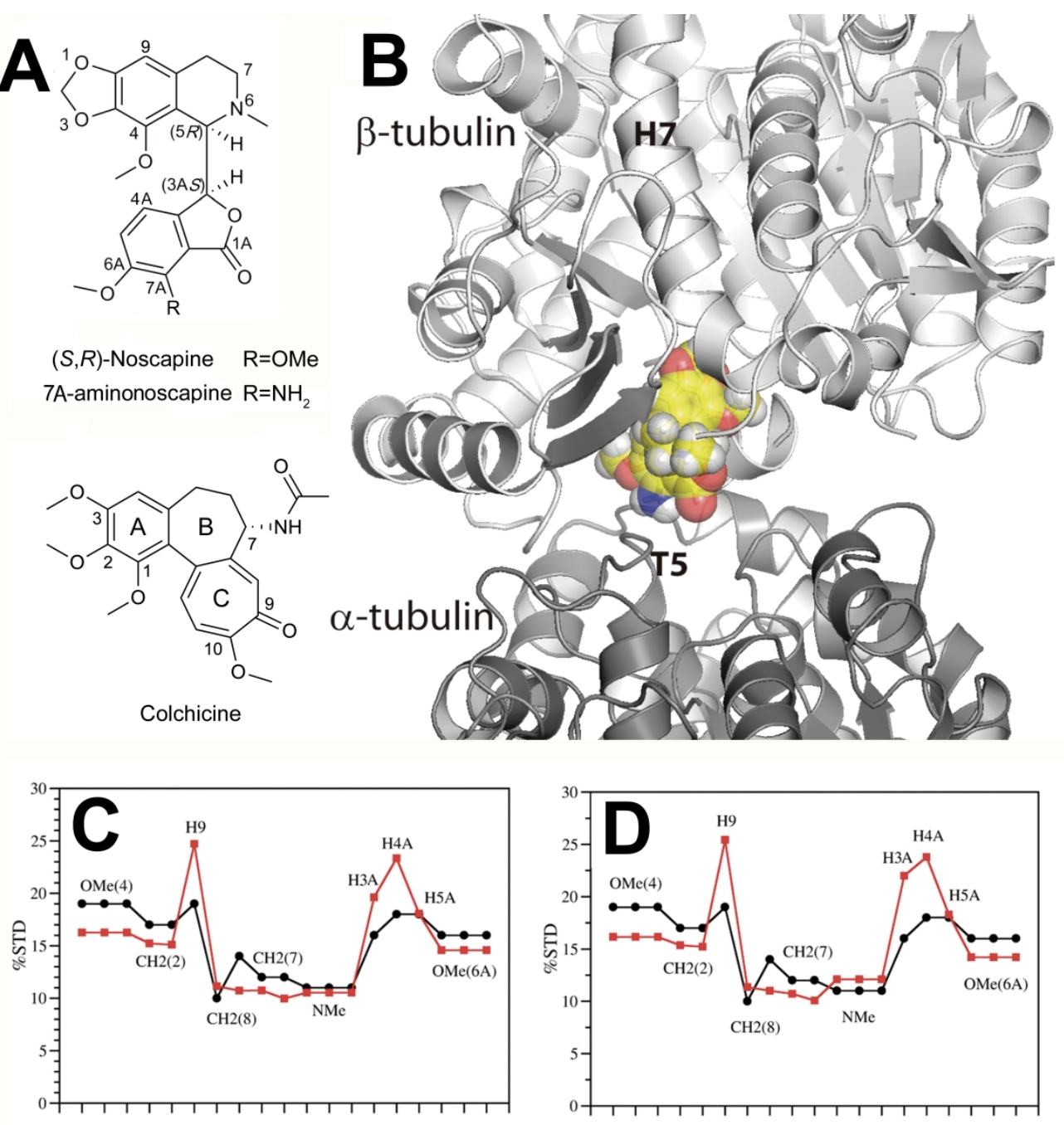

Figure 1.- A. Chemical structures of noscapine, 7A-aminonoscapine and colchicine. B. NMR calculated binding pose of 7A-aminonoscapine (yellow) at the colchicine site of $\beta 2$-tubulin of the T2R complex. C, D. Experimental STD profiles (black line) of the tubulin-7A-aminonoscapine complex as compared with STD profiles calculated (red line) from the generated binding poses in the $\beta 1-(C)$ and $\beta 2$-tubulin (D) subunits in the T2R-TTL complex. 

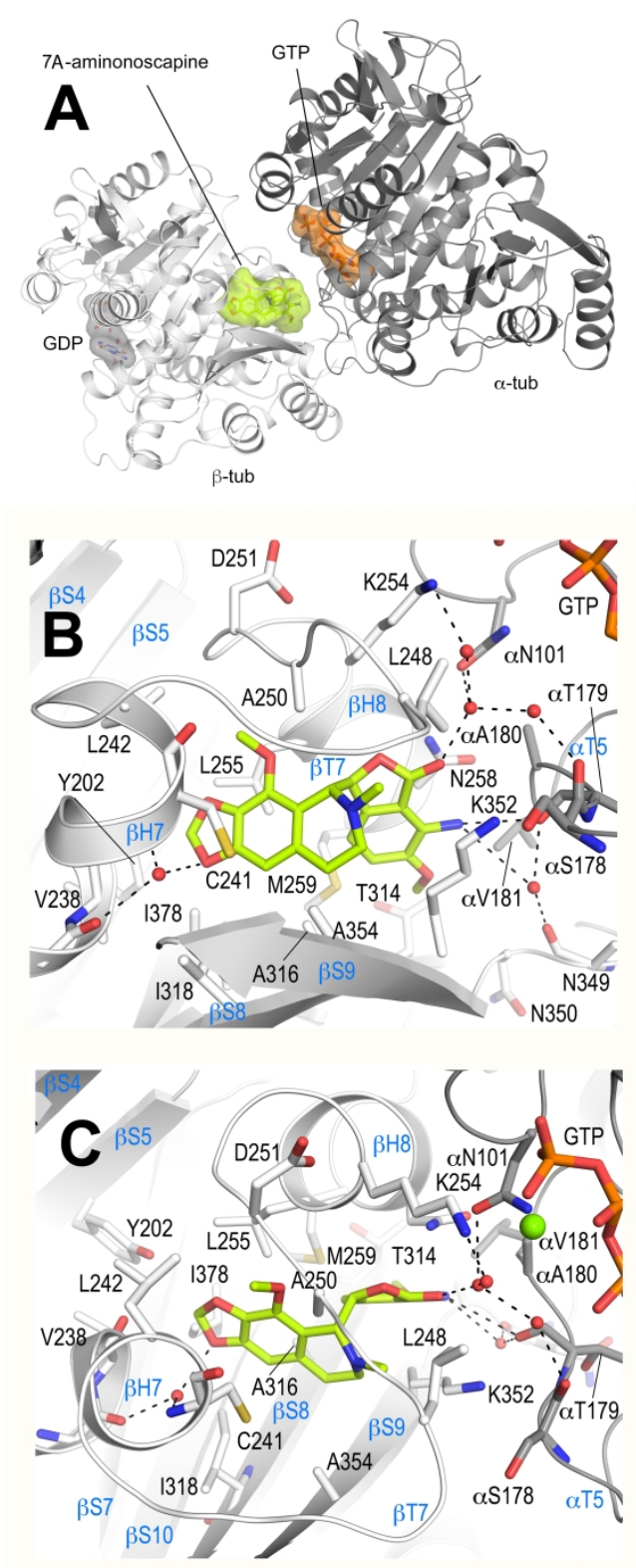

Figure 2.- Overall tubulin-7A-aminonoscapine complex structure. A. Ribbon representation of the tubulinbound 7A-aminonoscapine structure (PDB ID 6Y6D). The a- and $\beta$-tubulin chains are in dark and light grey, respectively. The ligand $7 A$-aminonoscapine (light green) and the nucleotides (orange) are in sphere and stick representation, respectively. The carbon atoms of the individual nucleotides are colored according to their chain assignments. Oxygen and nitrogen atoms are colored in red and blue, respectively. B, C. Closeup view of the atomic interaction network observed between 7A-aminonoscapine (light green) and tubulin (gray) in two different orientations. Interacting residues of tubulin are shown in stick representation and are labeled. Oxygen and nitrogen atoms are colored red and blue, respectively. Hydrogen bonds are depicted as black dashed lines. Secondary structural elements of tubulin are labeled in blue. For simplicity, only atubulin residues are indicated with a " $a$ ". 


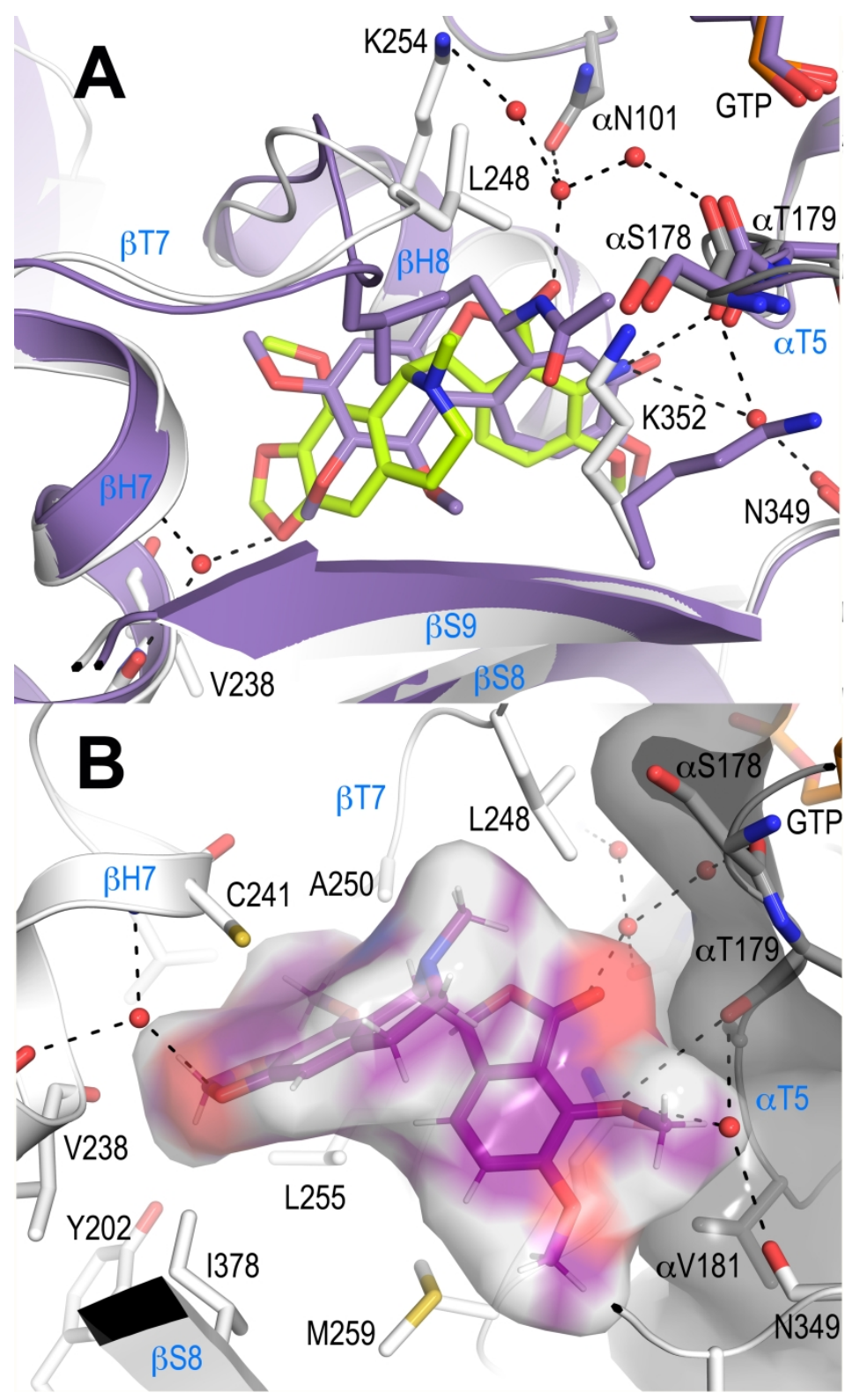

Figure 3.- A. Superposition of the tubulin-7A-aminonoscapine (light green, PDB ID 6Y6D) and tubulincolchicine (slate, PDB ID 4O2B) complex structures. The structures were superimposed onto the $\beta 1$-tubulin of their respective T2R-TTL complexes. Interacting residues of tubulin discussed in the text are shown in stick representation and are labeled. Oxygen and nitrogen atoms are colored red and blue, respectively. Hydrogen bonds are depicted as black dashed lines. Secondary structural elements of tubulin are labeled in blue. B. Modeled noscapine molecule into the crystallographic determined 7A-aminonoscapine binding site, highlighting how the 7A-methoxy group would clash into the aT5-loop. The clash is in agreement with the calculated NMR-model and suggests why noscapine does not bind to tubulin. 


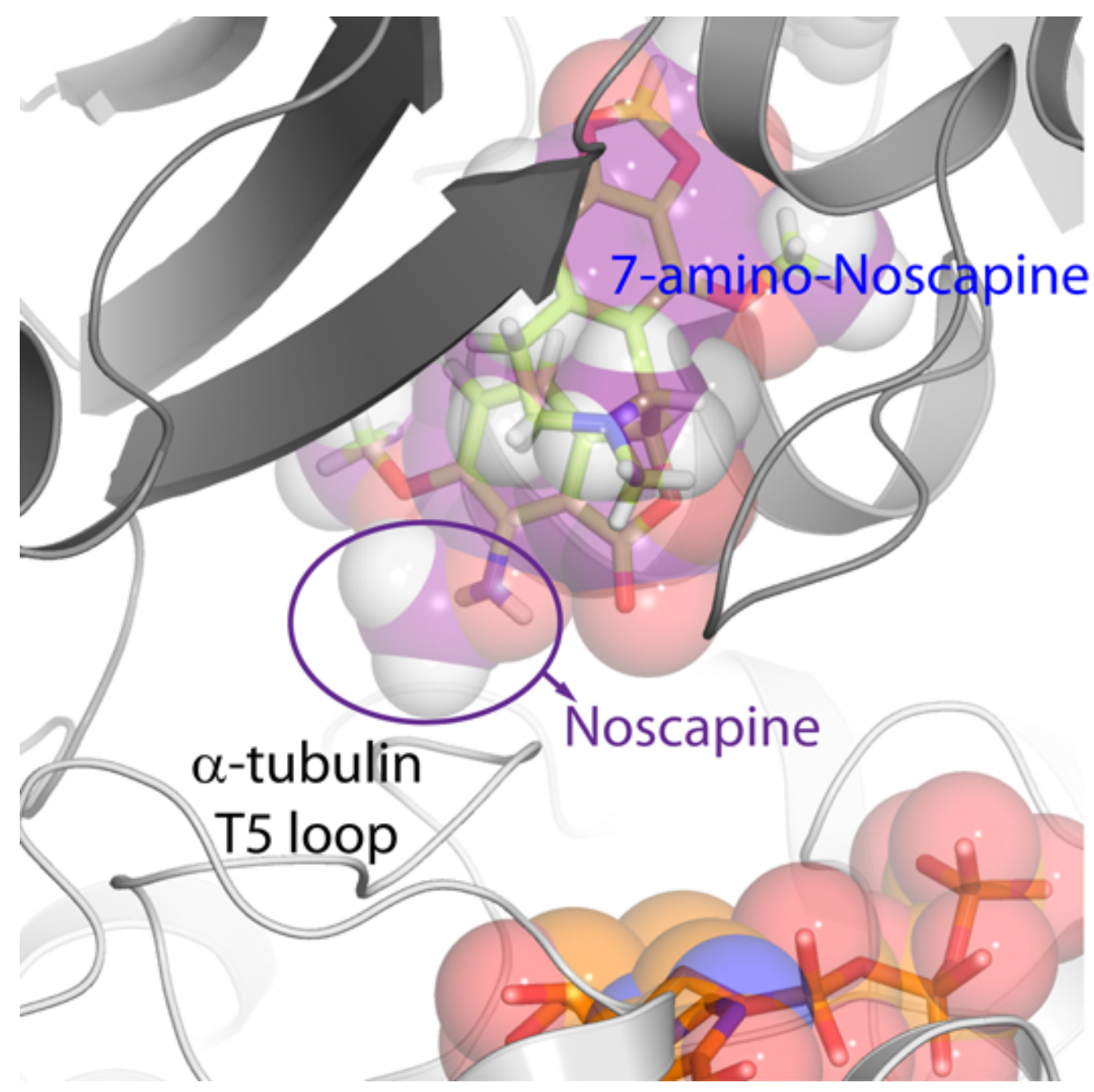

Table of Contents Grafic 